\section{OPEN ACCESS}

Edited by: David Della-Morte,

University of Miami, United States

Reviewed by:

Riccardo Lacchini,

University of São Paulo, Brazil

Andrea Marcia Marcaccini,

University of Ribeirão Preto, Brazil

*Correspondence:

Dao Pei Zhang

zhangdaopei89@163.com

Min Zhao

byts1969@126.com

tThese authors have contributed equally to this work

Specialty section:

This article was submitted to

Neuropharmacology,

a section of the journal

Frontiers in Neuroscience

Received: 22 May 2019

Accepted: 26 July 2019

Published: 14 August 2019

Citation:

Zhang DP, Peng YF, Zhang HL, Ma JG, Zhao M, Yin S and Wei TT (2019) Basilar Artery Tortuosity Is Associated With White Matter

Hyperintensities by TIMP-1.

Front. Neurosci. 13:836.

doi: 10.3389/fnins.2019.00836

\title{
Basilar Artery Tortuosity Is Associated With White Matter Hyperintensities by TIMP-1
}

\begin{abstract}
Dao Pei Zhang ${ }^{1 * t}$, Yan Fang Peng ${ }^{2 t}$, Huai Liang Zhang ${ }^{1}$, Jian Gong Ma ${ }^{1}$, Min Zhao ${ }^{1 *}$, Suo Yin ${ }^{3}$ and Tian Tian Wei ${ }^{4}$

'Department of Neurology, The First Affiliated Hospital of Henan University of Chinese Medicine, Zhengzhou, China, ${ }^{2}$ Department of Neurology, The Fifth Affiliated Hospital of Sun Yat-sen University, Zhuhai, China, ${ }^{3}$ Department of Image, People's Hospital of Zhengzhou Affiliated to Southern Medical University, Zhengzhou, China, ${ }^{4}$ Clinical Medical Testing Center, People's Hospital of Zhengzhou Affiliated to Southern Medical University, Zhengzhou, China
\end{abstract}

Background and Purpose: To test the hypothesis that the imbalance between matrix metalloproteinases (MMPs) and tissue inhibitor of metalloproteinases (TIMPs) may play a potential role in bridging vertebrobasilar dolichoectasia (VBD) with lacunar infarction (LI) and white matter hyperintensities (WMH).

Methods: We studied 212 patients with vertigo who underwent multimodal magnetic resonance imaging (MRI) tests for $\mathrm{VBD}, \mathrm{LI}$, and $\mathrm{WMH}$ identification. We investigated biomarkers of VBD with magnetic resonance angiography (MRA) via various physical characteristics of the vertebrobasilar arteries (VBAs). Similarly, LI and WMH biomarkers were extracted using T2-weighted and fluid attenuated inversion recovery (FLAIR) images. We first determined which of these neuroimaging markers were significant identifiers of $\mathrm{VBD}, \mathrm{LI}$ and the different grades of $\mathrm{WMH}$. We then sought to draw potential mechanistic conclusions from these MRI-derived parameters, by associating the aforementioned biomarkers with MMP and TIMP serum levels in patient blood samples using non-parametric statistical tests.

Results: MMP-9 serum level was significantly higher in vertigo patients with VBAs dilation and basilar artery (BA) elongation compared to those with healthy arterial size, and the ratio of MMP-9/TIMP-1 level were higher in those patients. TIMP-1 level was also markedly higher in vertigo patients with BA tortuosity than those without BA tortuosity. The bending length $(B L)$ of the BA was positively correlated with TIMP-1. The length, $B L$, and tortuosity index of the BA, as well as serum levels of TIMP-1 were greater in patients with higher WMH grades compared to those with low WMH grades. The vertebral artery and BA diameters, and the levels of MMP-2, $-3,-9$, TIMP-2 and cathepsin L were similar in patients with different $\mathrm{WMH}$ grades.

Conclusion: In vertigo patients, we found various probably associations between MMP-9 and TIMP-1 with arterial alterations linked to both VBD and WMH that may help with the diagnosis and treatment of such diseases in the future.

Keywords: matrix metalloproteinases, tissue inhibitor of metalloproteinase-1, vertebrobasilar dolichoectasia, cerebral small vessel disease, white matter hyperintensities 


\section{INTRODUCTION}

Vertebrobasilar dolichoectasia (VBD) is a clinical dysfunction characterized by an elongated, dilated and/or tortuous vertebral artery (VA) and/or basilar artery (BA) (Gutierrez et al., 2011; Yuan et al., 2014; Zhai et al., 2018). The prevalence of VBD is approximately $0.08-6.5 \%$ in the general population, while in patients with stroke, the prevalence ranges from 3 to $17 \%$ (Pico et al., 2015; Samim et al., 2016; Del Brutto et al., 2017). Similarly, cerebral small vessel disease (CSVD) is a heterogeneous dysfunction affecting the perforating cerebral arterioles, capillaries or venules. CSVD may manifest as acute focal neurological symptoms, dementia, gait disturbance and progressive cognitive impairment, however, the majority of patients are asymptomatic for large periods of time (Wardlaw et al., 2019). Nevertheless, while behavioral indicators may not be a reliable source of detecting VBD or CSVD, recent advances in magnetic resonance imaging (MRI) technology has led to the identification of various neuroimaging markers of both disease states (Pico et al., 2015; Del Brutto et al., 2017). The robustness and widespread accessibility of acquiring magnetic resonance angiography (MRA) information allows for simple quantification of vascular descriptors, such as the length and diameter of arteries linked to VBD. Alternatively, anatomybased MRI scans can identify lacunar infarcts (LI), white matter hyperintensities (WMH), dilated perivascular spaces, cerebral microbleeds and brain atrophy that are all regarded as indicators of CSVD (Norrving, 2015). Thus, in the current study, we employed various cutting-edge MRI techniques to identify and classify VBD and CSVD disease states in a large patient population.

It has been hypothesized that the involvement of matrix metalloproteinases (MMPs) and tissue inhibitor of metalloproteinases (TIMPs) play a meaningful role in both VBD and CSVD since both dysfunctions largely affect arterial media (Gutierrez et al., 2011; Pico et al., 2015; Del Brutto et al., 2017). In particular, VBD has been reported to be associated with elevated levels of MMP-9 expression alone or combined with low levels of TIMP-2 expression among individuals with HIV infection (Gutierrez et al., 2016). In a study using a mouse model focusing on differential inter-strain susceptibility to VBD, dolichoectasia was also reported to be associated with high MMP-12 and MMP-9 (Zhu et al., 2017). On the contrary, in a prospective cohort study of stroke patients, dolichoectasia was reported to be connected to a lower plasma level of MMP-3 (Pico et al., 2010). As for CSVD, a brain-autopsy study revealed that microglia/macrophage cells containing MMP-3 were more often present around small perforating arteries and areas of severe white matter damage corresponding to leukoaraiosis (Rosenberg et al., 2001). A recent study that genotyped MMP-2-1306 T/C and MMP-9-1562 C/T in a relatively large cohort of CSVD patients and controls came to a similar conclusion that MMP-2-1306 T/C polymorphism was associated with moderate or severe leukoaraiosis (Zhang et al., 2015). However, the mechanism regarding how the regulation of MMPs and TIMPs plays a role in the development and progression of VBD or CSVD remains poorly understood.
To investigate the role of MMPs and TIMPs in the pathogenesis of VBD and CSVD, we first used various MRI techniques to quantitatively identify significant neuroimaging biomarkers of these two neurological dysfunctions. We then extracted MMP and TIMP serum levels from blood samples to associate disease phenotypes found in the brain with the metabolic processes of the body. In this sense, we sought to draw mechanistic conclusions behind the dysfunctions of interest by associating MMP and TIMP serum levels with vertigo patients with and without VBD or CSVD.

\section{MATERIALS AND METHODS}

\section{Population}

The study was approved by the Ethics Committee of Zhengzhou People's Hospital and written informed consent was obtained from all participants. Data from 524 patients with vertigo, hospitalized at the Department of Neurology of Zhengzhou People's Hospital (China) from December 2014 to May 2017, were considered for participation.

According to a standard protocol, a trained neurologist (DZ) performed a neurological and vestibular examination on each patient. Most patients also underwent confirmatory caloric testing of vestibular function. Patients were included if they fulfilled the following criteria: (1) complaint of vertigo (main symptoms including spinning, swaying, nausea, vomiting and unsteady gait); (2) > 18 years old; (3) exhibit at least one vascular risk factor (see detailed description below); (4) had negative results in the Dix-Hallpike test and the Roll test. Patients were excluded if they reported/exhibited one of the following: benign paroxysmal positional vertigo (BPPV), Meniere's Disease, aural vertigo, medication/drug intoxication, recurrent ischemic strokes, atrial fibrillation or history of congenital heart disease. All patients were evaluated by CT scan $(n=524)$, MRI $(n=395)$ and/or MRA $(n=382)$. 146 patients were excluded due to encephalorrhagia $(n=4)$, the refusal to undergo MRI/MRA $(n=142)$, or the unavailability of MMP and TIMP data $(n=24)$. The 212 remaining patients with available MRI, MRA, MMPs and TIMPs participated in this study. A detailed roadmap for this recruiting process, as well as the analytical processes to compare the participating patients, is provided in Figure 1.

Various vascular risk factors were also examined and are as follows: history of arterial hypertension (previous diagnosis of arterial hypertension: systolic blood pressure $>160 \mathrm{mmHg}$, diastolic $>90 \mathrm{~mm} \mathrm{Hg}$, or past or present use of antihypertensive agents), diabetes mellitus (previous diagnosis of diabetes, or past or present use of antidiabetic agents), hyperlipidemia (cholesterol $>5.17 \mathrm{mmol} / \mathrm{L}$, triglycerides $>1.71 \mathrm{mmol} / \mathrm{L}$, or both), hyperhomocysteinemia ( $>15.0 \mu \mathrm{mol} / \mathrm{L}$ ), drinking at least once a week (one standard alcoholic beverage is equivalent to $120 \mathrm{~mL}$ of wine, $360 \mathrm{~mL}$ of beer, or $45 \mathrm{~mL}$ of distilled spirits), smoking (having continuously or cumulatively smoked for more than 6 months and at least one cigarette per day), moderate or severe intracranial/extracranial arterial stenosis (the degree of artery stenosis $>50 \%$ ) and history of coronary artery disease (CAD). All patients with vascular risk factors had been previously 


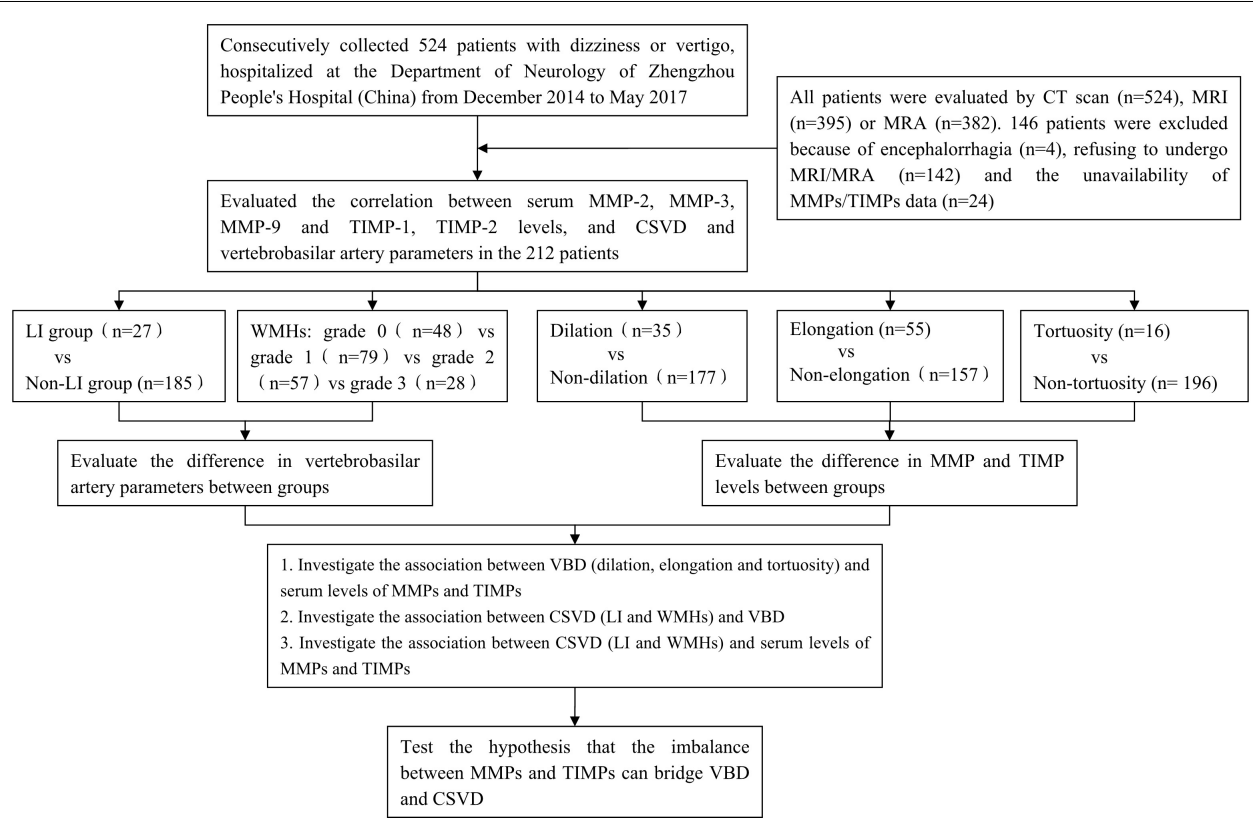

FIGURE 1 | Overall study roadmap. Flow chart detailing patient recruitment, parameter extraction, and analytical methods.

diagnosed as such and/or were already taking medications for their conditions.

\section{Assessment of Neuroimaging Markers of Vertebrobasilar Dolichoectasia}

Neuroimaging markers of VBD were evaluated using MRA images collected with a 3.0-T scanner (GE Medical, Piscataway, NJ, United States) within the first-week hospitalization. Three-dimensional time-of-flight (TOF) MRA was performed (repetition time $=24 \mathrm{~ms}$, echo time $=6 \mathrm{~ms}, \mathrm{FOV}=24 \times 24 \mathrm{~cm}$ and section thickness $=0.8-1.6 \mathrm{~mm}$ ) with image reconstruction using the maximum intensity projection.

Using these MRA images, we extracted the maximum diameter and tortuosity index (TI) of the BA and VAs, the basilar artery length (BAL) and the bending length (BL) of the BA. MRI analysis was performed by two experienced radiologists (SY and MY) blinded to clinical and demographic data. VA diameters were measured at three consecutive points, $3 \mathrm{~mm}$ apart, starting from the bilateral VA junction (only the maximum value was recorded for further analysis). The BA diameter was measured at the mid-pons level on TOF source images. BAL refers to a standard line length drawn from the top of the BA to the junction of both VAs (namely, the straightened length of BA), the actual length of BA was measured by tracing the course of the vessel from the top of the BA to the junction of both VAs. The BL refers to the vertical length between the midpoint of the width of the BA at the point of greatest bending and the standard line (Nishikata et al., 2004; Figure 2). For both VAs, the actual length was measured by tracing the course of the vessel from its origin to the vertebral level of $\mathrm{C} 2$, and the straightened length was calculated by measuring the linear distance from the origin to the end of the vessel (Morris et al., 2011; Figure 3). The TI of the BA

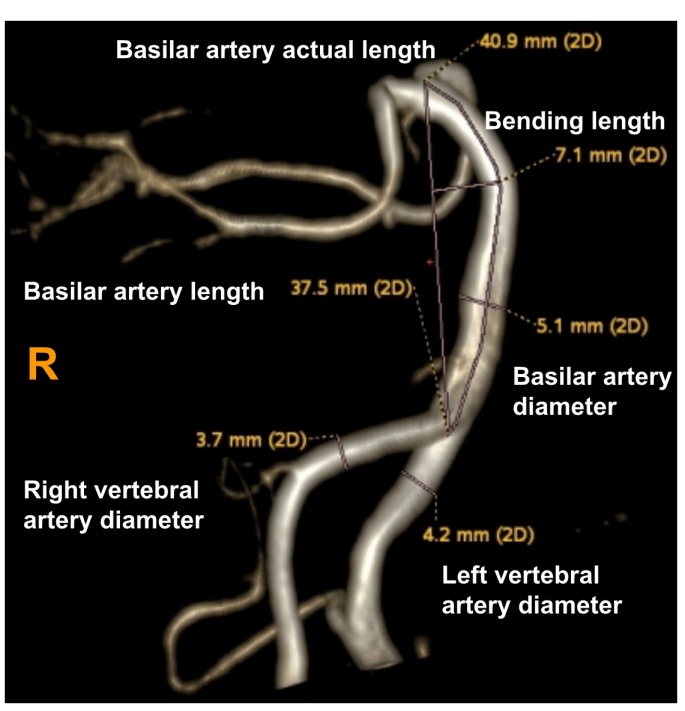

FIGURE 2 | Using a volume-rendered angiogram, the basilar artery tortuosity was measured in a vertebrobasilar dolichoectasia (VBD) patient. The picture shows that the right vertebral artery diameter is $3.7 \mathrm{~mm}$, left vertebral artery diameter is $4.2 \mathrm{~mm}$, basilar diameter is $5.1 \mathrm{~mm}$, basilar artery length (BAL) is $37.5 \mathrm{~mm}$, bending length (BL) is $7.1 \mathrm{~mm}$ and actual length of basilar artery is $40.9 \mathrm{~mm}$. Tortuosity index $(\mathrm{TI})$ of the $\mathrm{BA}=(40.9 / 37.5-1) \times 100=9.0$.

and VAs was defined according to Eq. 1.

$$
\left(\frac{\text { actual length }}{\text { Straightened length }}-1\right) \times 100
$$

Patients were classified as VBD if they met one or more of the following criteria: 


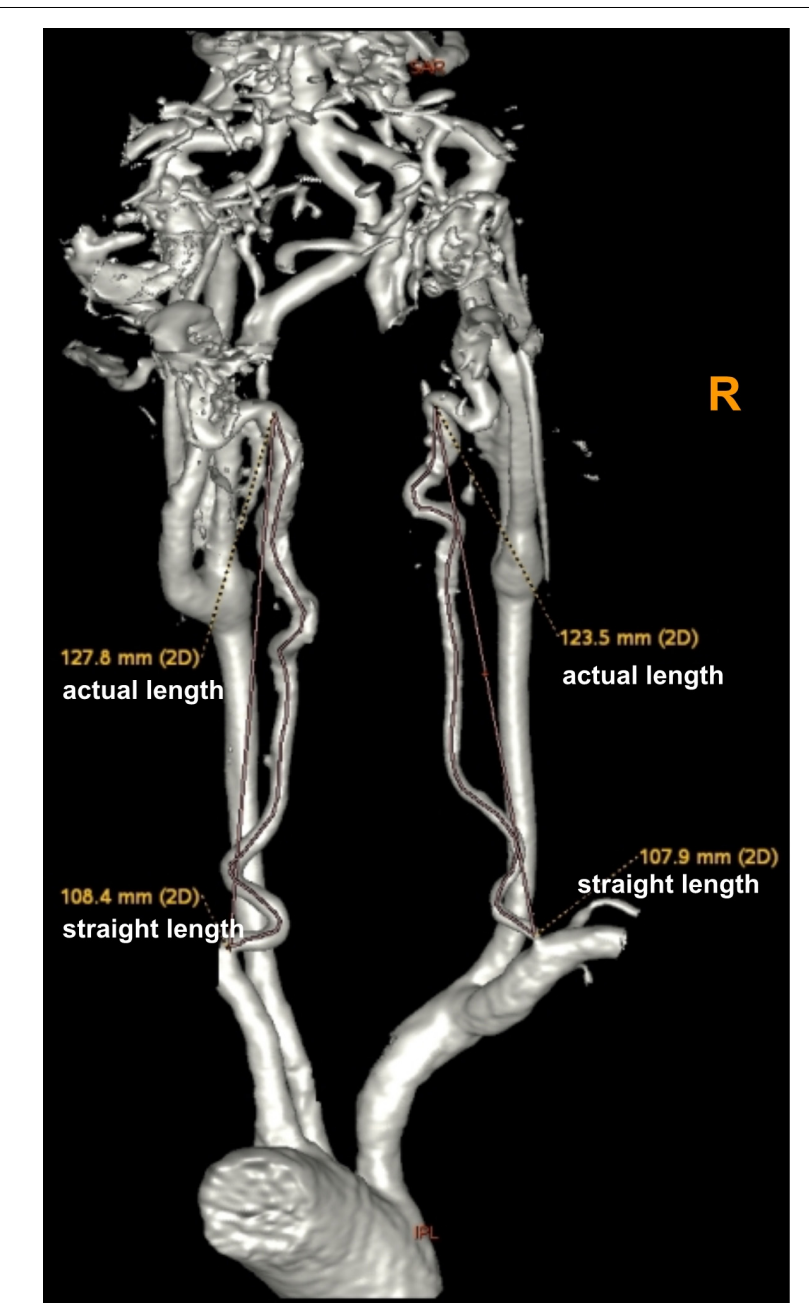

FIGURE 3 | Using a volume-rendered angiogram, the vertebral artery tortuosity was measured in a vertebrobasilar dolichoectasia (VBD) patient. The picture shows that the right vertebral artery actual length is $123.5 \mathrm{~mm}$, straightened length is $107.9 \mathrm{~mm}$, tortuosity index ( $\mathrm{TI})$ of the right $\mathrm{VA}=(123.5 / 107.9-1) \times 100=14.5$ and left vertebral artery actual length is $127.8 \mathrm{~mm}$, straightened length is $108.4 \mathrm{~mm}$, tortuosity index (TI) of the left $\mathrm{VA}=(127.8 / 108.4-1) \times 100=17.9$

(1) diameter of the $\mathrm{BA} \geq 4.5 \mathrm{~mm}$ or diameter of the VAs $\geq 4.0 \mathrm{~mm}$ (dilation) (Smoker et al., 1986; Zhai et al., 2018); (2) $\mathrm{BAL} \geq 29.5 \mathrm{~mm}$ (elongation) (Ubogu and Zaidat, 2004); (3) the $\mathrm{BL} \geq 8.0 \mathrm{~mm}$ (Thijs et al., 2017) (we also consider this condition as tortuous).

\section{Assessment of Neuroimaging Markers of Cerebral Small Vessel Disease}

Neuroimaging markers of CSVD were evaluated using conventional T2-weighted and fluid attenuated inversion recovery (FLAIR) anatomical images collected with a 3.0-T scanner (GE Medical, Piscataway, NJ, United States) within the first-week hospitalization. All images were obtained in the axial plane with voxels of size $1 \mathrm{~mm}$ wide $\times 1 \mathrm{~mm}$ high, $\times 5 \mathrm{~mm}$ thick $(\mathrm{TR}=4.8 \mathrm{~s}, \mathrm{TE}=6 \mathrm{~ms}$, field of view $320 \times 320 \mathrm{~mm})$.
CSVD was evaluated by two experienced radiologists blinded to clinical and demographic data, following the standards for reporting MRI-based vascular changes (Wardlaw et al., 2013). LI were defined as fluid-filled cavities in the basal ganglia, brain stem, or subcortical white matter with a diameter of 3 to $15 \mathrm{~mm}$. WMHs were defined as periventricular lesions appearing hyperintense on T2-weighted and FLAIR images without complete tissue destruction. WMHs were grouped into four grades according to the modified Fazekas scale $(0=$ absent; 1 = pencil-thin lining; $2=$ halo of $\geq 5 \mathrm{~mm}$ thickness; $3=$ irregular white matter hyperintensities extending into deep white matter) (Fazekas et al., 1987; Figure 4).

\section{Serological Test}

Venous blood samples were drawn within 1 week of hospital admission. Samples were allowed to clot for $30 \mathrm{~min}$ at room temperature in serum separator tubes (SST) before centrifugation for $15 \mathrm{~min}$ at $1000 \mathrm{~g}$. The plasma samples were frozen and stored at $\leq-80^{\circ} \mathrm{C}$ until further use.

Matrix metalloproteinases and TIMPs concentrations were tested using commercially available quantikine enzyme-linked immunosorbent assay (ELISA) kits (SMP300/SMMP200/SMP900/STM100/DTM200, R\&D Systems, Minneapolis, MN, United States). All samples were brought to room temperature before use and were analyzed in duplicate. The samples were diluted in the appropriate fold in assay dilution. A sample or standard was then added and incubated for $2 \mathrm{~h}$ at room temperature on a horizontal orbital microplate shaker $\left(0.12^{\prime \prime}\right.$ orbit $)$ set at $500 \pm 50 \mathrm{rpm}$. This was followed by incubation for $2 \mathrm{~h}$ with the following antibodies conjugates: total MMP-2, human MMP-3, human MMP-9, human TIMP-1 or human TIMP-2. Substrate solution was added to the samples and was incubated for $30 \mathrm{~min}$, after which a stop solution was added. The absorbance of color at $450 \mathrm{~nm}$ was measured using a microplate reader (Multiskan FC, Thermo) within 30 min. All steps were performed at room temperature (20$\left.25^{\circ} \mathrm{C}\right)$. All absorbance results are expressed as nanogram per milliliter $(n g / \mathrm{ml})$.

Cathepsin L concentration was detected with a DuoSet ELISA kit (DY008, R\&D Systems, Minneapolis, MN, United States). We sought to preprocess the microplates before running the assay procedure. Diluted capture antibody was coated to the microplate and incubated overnight. The plate was later blocked by incubating it with reagent diluent for a minimum of $1 \mathrm{~h}$. Since unblocked antibody was washed away, the plates were ready for sample addition. Samples or standards were added and incubated for $2 \mathrm{~h}$. The detection antibody and streptavidinhorseradish peroxidase were added in sequence and incubated for $20 \mathrm{~min}$. After adding substrate solution and allowing for $20 \mathrm{~min}$ of incubation, tetramethyl benzidine color liquid was added and at this point, a blue color developed in the microplate that was proportional to the amount of analyte present in the sample. Then, a stop solution was added, followed by gentle tapping to ensure thorough mixing, and the blue color turned to yellow. We determined the absorbance of the color immediately, using a microplate reader (Multiskan FC, Thermo) set to $450 \mathrm{~nm}$. All steps were performed at room 


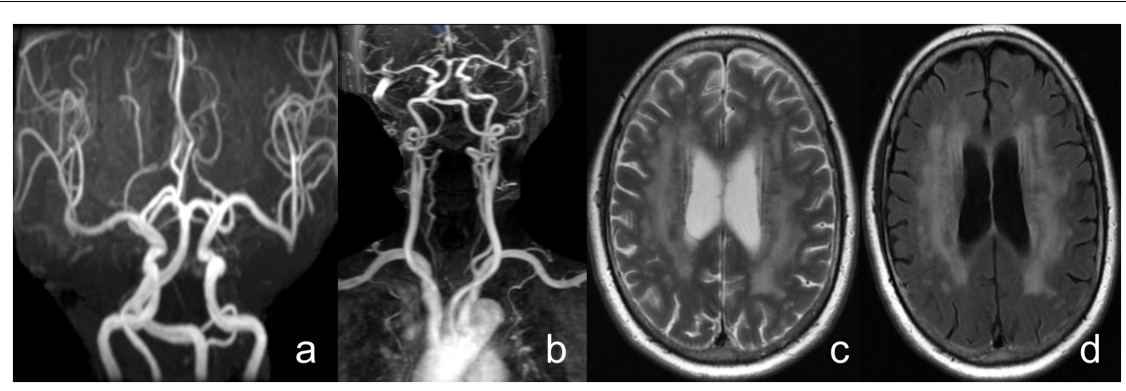

FIGURE 4 | A female patient, 71 years old, with complaints of "vertigo, nausea" and a history of hypertension. (a) Head MRA shows vertebrobasilar artery dolichoectasia, (b) Neck contrast enhanced MRA shows right vertebral artery dysplasia and bilateral vertebral artery tortuosity, (c,d) T2WI and FLAIR shows the side body of the lateral ventricle white matter high signal, Fazekas grade 3.

temperature $\left(20-25^{\circ} \mathrm{C}\right)$ and results are expressed as picogram per milliliter $(p g / \mathrm{ml})$.

\section{Statistical Analysis}

Data were analyzed with IBM SPSS version 20.0 and $P<0.05$ was considered significant. Demographic and clinical characteristics between VBD patients and non-VBD patients, and between CSVD patients and non-CSVD patients were respectively, evaluated by univariate analysis with a Student's $t$ test for continuous factors and a $\chi 2$ test for dichotomous factors (Fisher's exact test was used when the expected cell frequency was <5). Significant risk factors were accounted for in the multivariate analysis with adjustment for age and gender. MMPs and TIMPs serum levels between different VBD subclassifications (dilation vs. non-dilation group, tortuosity vs. non-tortuosity group, elongation vs. non-elongation group) were respectively, evaluated with a Mann-Whitney $U$ test. The correlation of serum MMPs and TIMPs levels and VBA parameters were calculated as the Pearson correlation coefficient. Testing of VBA parameters and serum MMPs and TIMPs levels between LI individuals and non-LI individuals was performed with a Mann-Whitney $U$ test, and between patients with different WMH grades with a KruskalWallis test.

\section{RESULTS}

\section{Demographic and Clinical Characteristics}

Of all 212 patients with available MRA and MMP data, the mean age was $60.2 \pm 12.5$ and $54 \%$ were male. In terms of the VBD imaging markers, 21 exhibited dilation, 32 elongation, 1 tortuosity, 8 dilation and elongation, 9 elongation and tortuosity, and 6 all of these. As for CSVD, we identified 27 patients with LI (18 lacunes and 9 multilacunar state) and 164 patients with $\mathrm{WMH}$. The classification of the periventricular lesions for $\mathrm{WMH}$ patients were as follows: 79 pencil-thin lining, 57 halo of $\geq 5 \mathrm{~mm}$ thickness, 28 irregular white matter hyperintensities extending into deep white matter. In vertigo patients with VBD, 43 patients were also diagnosed with CSVD, which was significantly different from those without $\operatorname{VBD}\left(\chi^{2}=8.033, P=0.018\right)$.
In the current study, age was found to be an independent risk factor of $\operatorname{VBD}(P=0.011)$, while gender, arterial hypertension, diabetes, dyslipidemia, hyperhomocysteinemia, smoking and alcoholism were not considered risks factors (all $P>0.0 .5$ ). Age, carotid arteries plaque and arterial hypertension were associated with CSVD whereas gender, diabetes, dyslipidemia, hyperhomocysteinemia, smoking and alcoholism were not associated with CSVD (Table 1).

\section{Serum MMP and TIMP Levels and Vertebrobasilar Arteries Parameters}

Dilation was defined as the diameter of the BA being greater than $4.5 \mathrm{~mm}$ or of the VAs being greater than $4.0 \mathrm{~mm}$. Patients exhibiting dilation (dilation group, $n=35$ ) were directly compared to those with healthy BA and VA diameters (non-dilation group, $n=177$ ). The MMP-9 serum level was significantly higher in the dilation group compared to the nondilation group $(P=0.021)$. However, there was no significant difference in MMP-2, -3, TIMP-1, -2 or cathepsin L serum level between two groups (all $P>0.05$ ). Further, the ratio of MMP2/TIMP-2 level was not significantly different between groups, however, the ratio of MMP-9/TIMP-1 level was in significant difference $(P=0.010$, Table 2$)$.

Basilar artery tortuosity was defined as the BL being great than $8.0 \mathrm{~mm}$; patients were divided into a tortuosity group $(n=16)$ or a non-tortuosity group $(n=196)$ based on this criterion. TIMP-1 serum level was markedly higher in the tortuosity group $(P=0.039)$ than the non-tortuosity group. Conversely, the level of MMP-2, -3, -9, TIMP-2 and cathepsin L serum was similar in the two groups (all $P>0.05$, Table 2). The ratio of MMP-2/TIMP-2 level or MMP-9/TIMP-1 level were neither significantly different between groups $(P>0.05$, Table 2$)$.

Patients were also divided into an elongation group $(n=55)$ or a non-elongation group $(n=157)$ according to the BAL. MMP9 serum level was significantly higher in the elongation group compared to the non-elongation group $(P=0.004)$. However, there was no significant difference in MMP-2, -3, TIMP-1, -2 or cathepsin L serum levels between two groups (all $P>0.05$, Table 2). The ratio of MMP-2/TIMP-2 level was not significantly different between groups, however, the ratio of MMP-9/TIMP-1 level was in significant difference $(P=0.016$, Table 2$)$. 
TABLE 1 | Demographic and clinical characteristics between VBD and non-VBD patients and between CSVD and non-CSVD individuals.

\begin{tabular}{|c|c|c|c|c|c|c|c|}
\hline & VBD $(n=77)$ & $\begin{array}{c}\text { Non-VBD } \\
(n=135)\end{array}$ & OR (95\% Cl) & $\begin{array}{l}\text { CSVD } \\
(n=170)\end{array}$ & Non-CSVD $(n=42)$ & $\begin{array}{l}\text { Unadjusted, OR } \\
(95 \% \mathrm{Cl})\end{array}$ & $\begin{array}{c}\text { Adjusted age and } \\
\text { gender, OR } \\
(95 \% \mathrm{Cl})\end{array}$ \\
\hline Age $(y)$, mean $\pm S D$ & $\begin{array}{c}64.12 \pm \\
12.014\end{array}$ & $\begin{array}{c}59.56 \pm \\
12.598\end{array}$ & $\begin{array}{c}1.769(-8.049 \\
-1.073) P=0.011\end{array}$ & $63.49 \pm 11.921$ & $52.00 \pm 10.813$ & $\begin{array}{c}2.018(-15.467 \\
-7.509)\end{array}$ & $\begin{array}{c}1.067(1.028 \\
1.107) P<0.01\end{array}$ \\
\hline Male, n (\%) & $44(57)$ & $70(52)$ & $\begin{array}{c}1.238(0.705 \\
2.175)\end{array}$ & $92(54)$ & $22(52)$ & $\begin{array}{l}1.072(0.545 \\
2.109)\end{array}$ & NS \\
\hline $\begin{array}{l}\text { Carotid arteries } \\
\text { plaques, n (\%) }\end{array}$ & $30(39)$ & 52 (39) & $\begin{array}{c}1.019(0.574 \\
1.809)\end{array}$ & $76(45)$ & $6(14)$ & $\begin{array}{c}4.851(1.942 \\
12.120) P<0.01\end{array}$ & $\begin{array}{c}3.544(1.307 \\
9.610) P<0.05\end{array}$ \\
\hline $\begin{array}{l}\text { Arterial hypertension, } n \\
(\%)\end{array}$ & $48(62)$ & $96(71)$ & $\begin{array}{c}0.672(0.372 \\
1.216)\end{array}$ & $129(76)$ & $15(36)$ & $\begin{array}{c}5.663(2.750 \\
11.663) P<0.01\end{array}$ & $\begin{array}{c}5.427(2.345 \\
12.559) P<0.01\end{array}$ \\
\hline Diabetes, n (\%) & $28(36)$ & $55(41)$ & $\begin{array}{c}0.831(0.467 \\
1.481)\end{array}$ & $73(43)$ & $10(24)$ & $\begin{array}{c}2.408(1.113 \\
5.213) P<0.05\end{array}$ & $\begin{array}{c}1.218(0.485 \\
3.054)\end{array}$ \\
\hline Dyslipidemia, n (\%) & $50(65)$ & $82(61)$ & $\begin{array}{c}1.197(0.669 \\
2.142)\end{array}$ & $103(61)$ & $29(69)$ & $\begin{array}{c}0.689(0.334 \\
1.420)\end{array}$ & \\
\hline CAD, n (\%) & $17(22)$ & $30(22)$ & $\begin{array}{c}0.992(0.505 \\
1.946)\end{array}$ & $40(24)$ & $7(17)$ & $\begin{array}{c}1.538(0.635 \\
3.730)\end{array}$ & \\
\hline $\begin{array}{l}\text { Hyperhomocysteinemia, } \\
\text { n (\%) }\end{array}$ & $27(35)$ & $35(26)$ & $\begin{array}{c}1.543(0.842 \\
2.828)\end{array}$ & $56(33)$ & $6(14)$ & $\begin{array}{c}2.947(1.173 \\
7.407) P<0.05\end{array}$ & $\begin{array}{c}2.683 \\
(0.932,7.724)\end{array}$ \\
\hline Hyperuricemia, n (\%) & $3(4)$ & $6(4)$ & $\begin{array}{c}0.872(0.212 \\
3.588)\end{array}$ & $7(4)$ & $2(5)$ & $\begin{array}{c}0.859(0.172 \\
4.293)\end{array}$ & \\
\hline Smoking, n (\%) & $23(30)$ & $36(27)$ & $\begin{array}{l}1.171(0.630 \\
2.176)\end{array}$ & $44(26)$ & $15(36)$ & $\begin{array}{c}0.629(0.306 \\
1.289)\end{array}$ & \\
\hline Alcoholism, n (\%) & $12(16)$ & $25(19)$ & $\begin{array}{c}0.812(0.382 \\
1.726)\end{array}$ & $31(18)$ & $6(14)$ & $\begin{array}{c}1.338(0.519 \\
3.453)\end{array}$ & \\
\hline $\begin{array}{l}\text { Moderate or severe } \\
\text { intracranial/extracranial } \\
\text { arterial stenosis, n (\%) }\end{array}$ & $5(6)$ & $18(13)$ & $\begin{array}{c}0.451(0.161 \\
1.269)\end{array}$ & $20(12)$ & $3(7)$ & $\begin{array}{c}1.733(0.490 \\
6.133)\end{array}$ & \\
\hline
\end{tabular}

VBD indicates vertebrobasilar dolichoectasia; CSVD, cerebral small vessel disease; and CAD, coronary artery disease.

We further calculated the correlation between serum MMPs and TIMPs levels and VA parameters. We found that the maximum diameter of the right VA was negatively correlated with MMP-2 serum level $(r=-0138, P=0.046)$ and positively correlated with MMP-9 serum level $(r=0.164, P=0.018)$. Furthermore, TI was positively correlated with TIMP-1 serum level $(r=0.142, P=0.041)$. The diameter or TI of the left VA was not correlated with any MMP or TIMP levels. Interestingly, we found that the BL of the BA was positively correlated with TIMP-1 serum level (Figure 5) $(r=0.161, P=0.020)$ though the diameter, BAL and TI of the BA were not correlated with any MMP or TIMP serum level. The parameters of VAs and BA were not correlated with the ratio of MMP-2/TIMP-2 level or MMP-9/TIMP-1 level $(P>0.05)$.

\section{Vertebrobasilar Arteries Parameters and the Neuroimaging Markers of Cerebral Small Vessel Disease}

To investigate the association between VBD and CSVD, neuroimaging parameters including the diameters and TI of the VAs and BA, as well as the BAL and BL of the BA were evaluated between vertigo patients with or without $\mathrm{LI}$ and among different grades of WMH. The diameter and TI of the VAs and BA, and the BAL and BL of the BA were all similar in vertigo patients with LI $(n=27)$ and those without LI $(n=185)$ (Table 3$)$. The diameter of the VAs and BA were similar in vertigo patients with different WMH grades. Interestingly though, the BAL, TI and BL were significantly greater (all $P<0.01$ ) in vertigo patients with higher WMH grades (grade 2 and 3$)(n=85)$ than those with lower WMH grades (grade 0 and 1) $(n=127)$ (Table 3). We also found that the TI of the VAs were nearly significantly greater (both $P<0.20$ ) in high grade WMH patients compared to low grade $\mathrm{WMH}$ patients (Table 3 ).

\section{Serum MMP and TIMP Levels and the Neuroimaging Markers of Cerebral Small Vessel Disease}

The neuroimaging markers of CSVD, including LI and WMH, were observed in the current study. There was no significant difference in the serum level of MMP-2, -3, -9, TIMP-2 or cathepsin L, or the ratio of MMP-2/TIMP-2 level or MMP9/TIMP-1 level between vertigo patients with LI $(n=27)$ and those without LI $(n=185)$. We did, however, identify a near significant difference in the TIMP-1 serum level, being higher in vertigo patients with $\mathrm{LI}$ compared to those without $\mathrm{LI}(P<0.10$, Table 3). On the other hand, the TIMP-1 serum level was markedly higher in vertigo patients with high grade $\mathrm{WMH}$ (grade 2 and 3) $(n=85)$ than those with low grade WMH (grade 0 and grade 1$)(n=127)(P<0.01)$. There was no significant difference in the serum level of MMP-2, $-3,-9$, TIMP-2 or cathepsin L, or the ratio of MMP-2/TIMP-2 level or MMP-9/TIMP-1 level among individuals with different WMH grades (Table 3 ). 


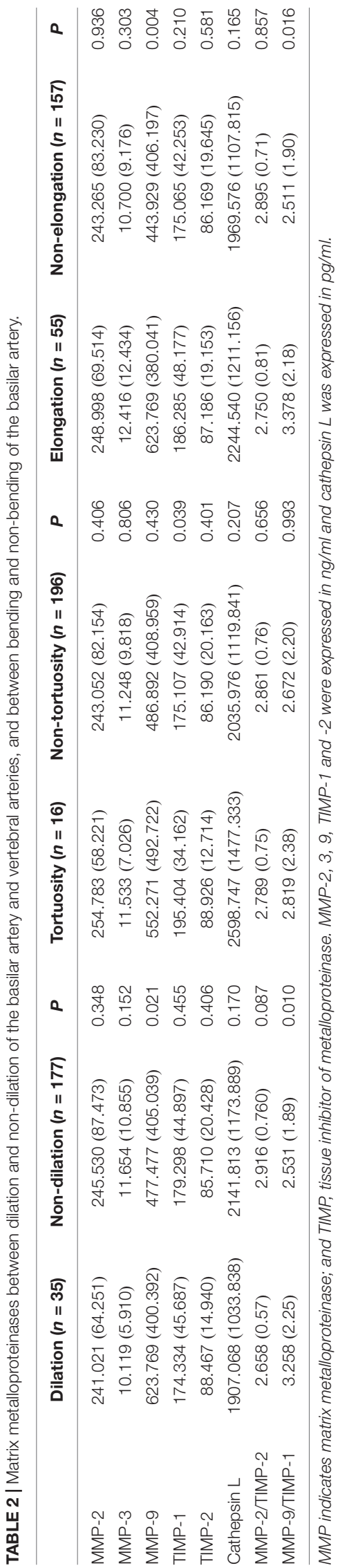

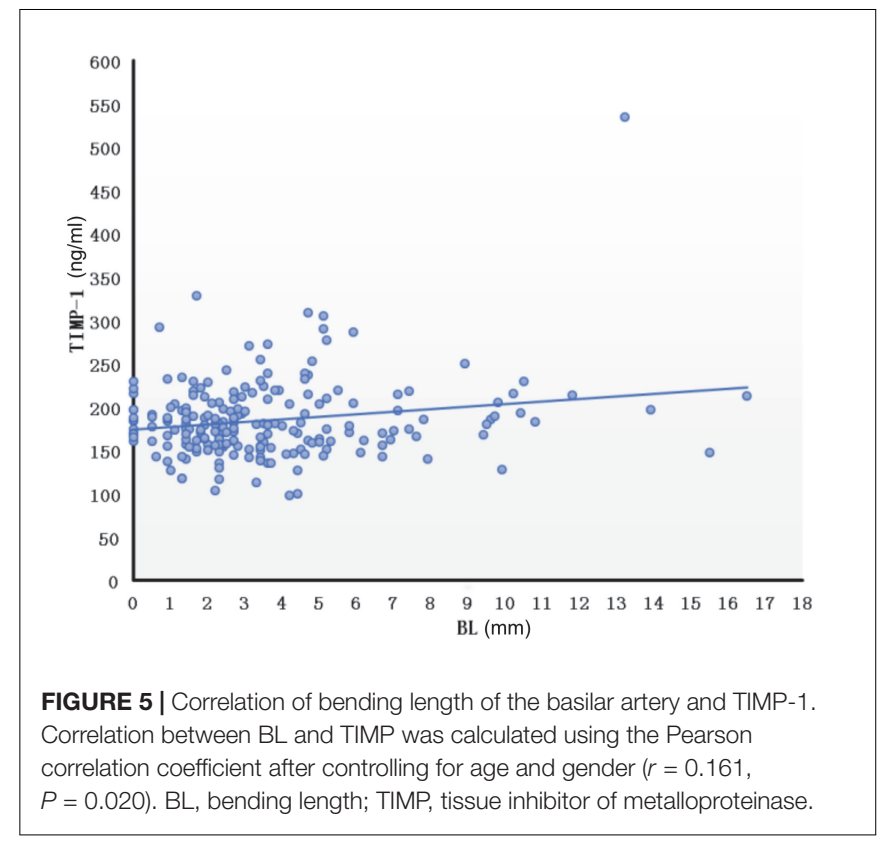

\section{DISCUSSION}

The relationships between VBD and the imbalance of MMPs and TIMPs have been speculated for years, but it has not been clarified how the imbalance between MMPs and TIMPs play a role in the development and progression of dolichoectasia (Gutierrez et al., 2011; Pico et al., 2015). We found that MMP-9 serum level was higher in vertigo patients with VBA dilation and BA elongation, and the ratio of MMP9/TIMP-1 level were higher in those patients, while TIMP-1 serum level was higher in those patients with BA tortuosity. The BL, which represents the degree of BA tortuosity, was positively correlated with TIMP-1. MMP-9 and TIMP-1 serum levels were both higher in vertigo patients with VBD, and we further found that upregulation of MMP-9 might be accompanied by the downregulation of TIMP- 1 but not by TIMP2 regulation.

Matrix metalloproteinases and TIMPs maintain a relative balance in normal tissues (Cui et al., 2017) and as this balance is disturbed, the degradation of extracellular matrix occurs. A recent study on patients with Graves' orbitopathy unfolded that MMP-2, MMP-9, TIMP-1 and TIMP-2 serum concentrations were all significantly higher than healthy controls (KapelkoSłowik et al., 2018). In addition, another study on multiple myeloma patients revealed that MMP-2 and MMP-9 are secreted in higher amounts and are not balanced by inhibitors of TIMPs (Urbaniak-Kujda et al., 2016). Therefore, growing evidence supports the phenomenon that MMPs and TIMPs maintain a balance in a normal state, and when broken, both MMPs and TIMPs are regulated via a positive feedback loop. For example, increasing MMPs breaks the balance between MMPs and TIMPs, and the body compensates by increasing TIMPs via a positive feedback mechanism. However, if the MMP increase surpasses 
TABLE 3 | Association between cerebral small vessel disease and vertebrobasilar artery parameters and MMPs.

\begin{tabular}{|c|c|c|c|c|c|c|c|c|}
\hline & \multirow[t]{2}{*}{ 니 $(n=27)$} & \multirow[t]{2}{*}{ Non-LI $(n=185)$} & \multirow[t]{2}{*}{$P$} & \multicolumn{4}{|c|}{ WMH } & \multirow[t]{2}{*}{$P$} \\
\hline & & & & Grade $0(n=48)$ & Grade $1(n=79)$ & Grade $2(n=57)$ & Grade $3(n=28)$ & \\
\hline \multicolumn{9}{|c|}{$\begin{array}{l}\text { Vertebrobasilar } \\
\text { arteries parameters }\end{array}$} \\
\hline \multicolumn{9}{|c|}{ Left vertebral artery } \\
\hline Diameter & $2.90(1.60)$ & $2.90(1.20)$ & 0.668 & $3.10(1.20)$ & $3.15(1.20)$ & $2.80(1.30)$ & $2.80(1.50)$ & 0.791 \\
\hline $\mathrm{Tl}$ & $0.958(0.105)$ & $0.967(0.099)$ & 0.514 & $0.769(0.112)$ & $0.101(0.094)$ & $0.103(0.104)$ & $1.116(0.105)$ & 0.072 \\
\hline \multicolumn{9}{|c|}{ Right vertebral artery } \\
\hline Diameter & $2.90(1.70)$ & $2.50(1.20)$ & 0.078 & $2.45(1.30)$ & $2.50(1.40)$ & $2.60(1.20)$ & $2.75(1.30)$ & 0.403 \\
\hline $\mathrm{Tl}$ & 0.739 (0.038) & $0.657(0.061)$ & 0.369 & $0.050(0.060)$ & $0.067(0.044)$ & $0.070(0.064)$ & $0.068(0.053)$ & 0.161 \\
\hline \multicolumn{9}{|l|}{ Basilar artery } \\
\hline Diameter & $3.37(0.90)$ & $3.27(0.80)$ & 0.285 & $3.15(0.90)$ & $3.35(0.80)$ & $3.30(0.70)$ & $3.35(1.40)$ & 0.631 \\
\hline BAL & $26.30(6.80)$ & $26.35(6.10)$ & 0.490 & $26.25(5.30)$ & $25.55(6.00)$ & $26.70(8.40)$ & $30.85(11.10)$ & 0.000 \\
\hline $\mathrm{Tl}$ & $0.056(0.100)$ & $0.045(0.087)$ & 0.362 & $0.054(0.055)$ & $0.031(0.071)$ & $0.042(0.126)$ & $0.112(0.200)$ & 0.001 \\
\hline $\mathrm{BL}$ & $3.40(3.70)$ & $2.70(3.00)$ & 0.493 & $2.85(2.70)$ & $2.30(2.20)$ & $2.60(3.60)$ & $4.65(6.90)$ & 0.000 \\
\hline \multicolumn{9}{|c|}{ MMPs parameters } \\
\hline MMP-2 & 261.736 (74.064) & 242.879 (82.154) & 0.421 & $243.545(100.032)$ & $242.879(81.825)$ & 257.069 (85.350) & 242.646 (39.044) & 0.769 \\
\hline MMP-3 & $12.728(15.328)$ & 11.195 (8.982) & 0.294 & $12.041(11.551)$ & 9.924 (8.861) & $11.713(10.967)$ & $10.564(7.465)$ & 0.388 \\
\hline MMP-9 & 564.603 (479.015) & $481.822(389.665)$ & 0.323 & $428.596(389.421)$ & $496.223(496.223)$ & $562.006(394.830)$ & 514.535 (363.744) & 0.392 \\
\hline TIMP-1 & 189.834 (52.559) & $175.107(45.541)$ & 0.098 & 161.035 (37.367) & $175.461(41.415)$ & $184.360(39.317)$ & 187.950 (62.638) & 0.008 \\
\hline TIMP-2 & 89.7129 (16.449) & 85.960 (20.039) & 0.507 & 89.676 (19.469) & 84.368 (23.227) & 88.467 (17.197) & 86.698 (14.788) & 0.622 \\
\hline Cathepsin L & $2244.540(1070.235)$ & 2030.142 (1177.967) & 0.178 & $1830.532(903.881)$ & 2175.154 (1213.089) & 2236.315 (1340.341) & $1960.427(1356.551)$ & 0.150 \\
\hline MMP-2/TIMP-2 & $2.895(0.70)$ & $2.845(0.73)$ & 0.784 & $2.843(0.72)$ & $2.910(0.84)$ & $2.916(0.65)$ & $2.734(0.76)$ & 0.946 \\
\hline MMP-9/TIMP-1 & $3.091(2.62)$ & 2.668 (2.19) & 0.558 & $2.486(2.33)$ & $2.668(2.46)$ & $2.695(2.17)$ & $2.819(1.70)$ & 0.872 \\
\hline
\end{tabular}

LI indicates lacunar infarction; WMH, white matter hyperintensities; TI, tortuosity index; BAL, basilar artery length; BL, bending length; MMP, matrix metalloproteinase; and TIMP, tissue inhibitor of metalloproteinase. 
the capability of such regulation, increasing TIMPs is inadequate to regain balance with MMPs. This can lead to the degradation of extracellular proteins located in the tunica media, such as elastin, collagen, or proteoglycans, leading to the procession of artery dilation (Pico et al., 2015). Nevertheless, how excessive increases of TIMPs lead to the dolichoectasia of arteries remains unclear. In the current study, MMP-9 and TIMP-1 serum levels were both higher in patients with VBD, and the ratio of MMP9/TIMP-1 was higher in patients with vertebrobasilar dilation and elongation, which was likely to add the evidence that increasing MMP-9 beaks the balance between MMP9 and TIMP-1 leading to the dilation or elongation of arteries. However, the interesting phenomenon of the balance between MMPs and TIMPs in vertigo patients with VBD should be further evaluated in future study, especially evaluating an animal model with genetic or pharmacological tools, to elucidate a deeper understanding of pathophysiological VBD.

We also found that CSVD was more common in vertigo patients with VBD than those without VBD. Very recent studies have revealed that CSVD is frequent in stroke patients with intracranial arteries dolichoectasia (Thijs et al., 2017; Zhai et al., 2018; Fierini et al., 2019). Importantly, we evaluated VBD with quantitative image parameters, and found that $\mathrm{BAL}$, and the BL and the TI of the BA were markedly greater (and the TI of the VAs to a lesser extent) in vertigo patients with higher WMH grades (grade 2 and 3 ) compared to those with lower WMH grades (grade 0 and 1). Similarly, a new report (Thijs et al., 2017) also found that dolichoectasia was associated with higher grades of $\mathrm{WMH}$, however, they didn't assess the degree of VBD. CSVD is an arteriopathy affecting the media of the artery as well, and it is associated with MMP and TIMP levels (Rosenberg et al., 2001; Zhang et al., 2015; Del Brutto et al., 2017). A study discovered that microglia/macrophages cells contained MMP-3 were more present around small perforating arteries and areas of severe white matter damage corresponding to leukoaraiosis (Rosenberg et al., 2001). Another study concluded that MMP2-1306 T/C polymorphism was associated with moderate or severe leukoaraiosis (Zhang et al., 2015). However, in the current study, MMP-2, -3 or -9 levels were not higher in vertigo patients with LI or advanced stages of $\mathrm{WMH}$, whereas the TIMP-1 level was higher in vertigo patients with higher WMH grades (grade 2 and 3). Our results, to some extent, support the hypothesis that the imbalance between MMPs and TIMPs could be a bridge between VBD and WMH. However, in order to provide mechanistic evidences, an animal model with genetic or pharmacological tools should be well performed in a further series of studies to provide hard evidence of the causality relationship among MMPs, TIMPs, VBD and WMH.

Some limitations in our study need consideration. First, this study was a cross-sectional study in a single center and asymptomatic patients were excluded. Thus, our data were limited, and a selection bias might be unavoidable. Second, recognizing the dilated perivascular spaces was subjective and may have led to large heterogeneity across patients. Furthermore, there were just three participating patients that underwent susceptibility-weighted imaging to detect cerebral microbleeds, and we failed to evaluate these neuroimaging markers of CSVD in our study. Third, we evaluated MMP and TIMP serum levels but never performed the pathological examination to evaluate the local concentrations of MMPs and TIMPs in the VBAs or in the areas of LI or WMH.

\section{CONCLUSION}

In conclusions, an imbalance between MMP-9 and TIMP1 levels probably link the extent of VBD and $\mathrm{WMH}$ in the current study. An upregulation of MMP-9 mainly might result in VBA dilation of the VAs and elongation of the BA while an upregulation of TIMP-1 mainly might lead to BA tortuosity. This BA tortuosity likely contributes to the occurrence and progression of WMH. Future studies will expand the sample size and conduct regular patient follow-ups to test our hypothesis. An animal model with genetic or pharmacological tools also should be evaluated to provide hard evidence on the association among VBD, CSVD and MMPs and TIMPs.

\section{DATA AVAILABILITY}

All datasets generated for this study are included in the manuscript and/or the supplementary files.

\section{AUTHOR CONTRIBUTIONS}

$\mathrm{DZ}$ and MZ conceived this study and provided financial support. YP analyzed the whole data and wrote the draft manuscript. HZ and JG collected the clinical data of the patients. SY collected and analyzed the image data. TW tested the serum levels of MMPs and TIMPs.

\section{FUNDING}

This study was supported by the National Natural Science Foundation of China (Grant No. 81471203).

\section{ACKNOWLEDGMENTS}

We are grateful to Mei Yun Wang (Department of Neuroimaging, People's Hospital Affiliated to Zhengzhou University) for her technical assistance. 


\section{REFERENCES}

Cui, N., Hu, M., and Khalil, R. A. (2017). Biochemical and biological attributes of matrix metalloproteinases. Prog. Mol. Biol. Transl. Sci. 147, 1-73. doi: 10.1016/ bs.pmbts.2017.02.005

Del Brutto, V. J., Ortiz, J. G., and Biller, J. (2017). Intracranial arterial dolichoectasia. Front. Neurol. 17:344. doi: 10.3389/fneur.2017.00344

Fazekas, F., Chawluk, J. B., Alavi, A., Hurtig, H. I., and Zimmerman, R. A. (1987). MR signal abnormalities at $1.5 \mathrm{~T}$ in Alzheimer's dementia and normal aging. AJR Am. J. Roentgenol. 149, 351-356. doi: 10.2214/ajr.149.2.351

Fierini, F., Poggesi, A., Salvadori, E., Acquafresca, M., Fainardi, E., Moretti, M., et al. (2019). Cerebral small vessel disease and systemic arteriopathy in intracranial arterial dolichoectasia patients. Acta Neurol. Scand. 139, 150-157. doi: 10.1111/ane.13038

Gutierrez, J., Menshawy, K., Goldman, J., Dwork, A. J., Elkind, M. S., Marshall, R. S., et al. (2016). Metalloproteinases and brain arterial remodeling among individuals with and those without HIV infection. J. Infect. Dis. 214, 1329-1335. doi: 10.1093/infdis/jiw385

Gutierrez, J., Sacco, R. L., and Wright, C. B. (2011). Dolichoectasia-an evolving arterial disease. Nat. Rev. Neurol. 7, 41-50. doi: 10.1038/nrneurol.2010.181

Kapelko-Słowik, K., Słowik, M., Szaliński, M., Dybko, J., Wołowiec, D., Prajs, I., et al. (2018). Elevated serum concentrations of metalloproteinases (MMP2, MMP-9) and their inhibitors (TIMP-1, TIMP-2) in patients with Graves' orbitopathy. Adv. Clin. Exp. Med. 27, 99-103. doi: 10.17219/acem/68991

Morris, S. A., Orbach, D. B., Geva, T., Singh, M. N., Gauvreau, K., and Lacro, R. V. (2011). Increased vertebral artery tortuosity index is associated with adverse outcomes in children and young adults with connective tissue disorders. Circulation 124, 388-396. doi: 10.1161/CIRCULATIONAHA.110.99 0549

Nishikata, M., Hirashima, Y., Tomita, T., Futatsuya, R., Horie, Y., and Endo, S. (2004). Measurement of basilar artery bending and elongation by magnetic resonance cerebral angiography: relationship to age, sex and vertebral artery dominance. Arch. Gerontol. Geriatr. 38, 251-259. doi: 10.1016/j.archger.2003. 10.006

Norrving, B. (2015). Evolving concept of small vessel disease through advanced brain imaging. J. Stroke 17, 94-100. doi: 10.5853/jos.2015.17.2.94

Pico, F., Jacob, M. P., Labreuche, J., Soufir, N., Touboul, P. J., Benessiano, J., et al. (2010). Matrix metalloproteinase-3 and intracranial arterial dolichoectasia. Ann. Neurol. 67, 508-515. doi: 10.1002/ana.21922

Pico, F., Labreuche, J., and Amarenco, P. (2015). Pathophysiology, presentation, prognosis, and management of intracranial arterial dolichoectasia. Lancet Neurol. 14, 833-845. doi: 10.1016/S1474-4422(15)00089-7

Rosenberg, G. A., Sullivan, N., and Esiri, M. M. (2001). White matter damage is associated with matrix metalloproteinases in vascular dementia. Stroke 32, 1162-1168. doi: 10.1161/01.str.32.5.1162

Samim, M., Goldstein, A., Schindler, J., and Johnson, M. H. (2016). Multimodality imaging of vertebrobasilar dolichoectasia: clinical presentations and imaging spectrum. Radiographics 36, 1129-1146. doi: 10.1148/rg.2016150032
Smoker, W. R., Price, M. J., Keyes, W. D., Corbett, J. J., and Gentry, L. R. (1986). High-resolution computed tomography of the basilar artery: 1. Normal size and position. AJNR Am. J. Neuroradiol. 7, 55-60.

Thijs, V., Grittner, U., Fazekas, F., McCabe, D. J. H., Giese, A. K., Kessler, C., et al. (2017). Dolichoectasia and small vessel disease in young patients with transient ischemic attack and stroke. Stroke 48, 2361-2367. doi: 10.1161/STROKEAHA. 117.017406

Ubogu, E. E., and Zaidat, O. O. (2004). Vertebrobasilar dolichoectasia diagnosed by maganetic resonance angiography and risk of stroke and death: a cohort study. J. Neurol. Neurosurg. Psychiatry 75, 22-26.

Urbaniak-Kujda, D., Kapelko-Slowik, K., Prajs, I., Dybko, J., Wolowiec, D., Biernat, M., et al. (2016). Increased expression of metalloproteinase-2 and -9 (MMP2, MMP-9), tissue inhibitor of metalloproteinase-1 and -2 (TIMP-1, TIMP-2), and EMMPRIN (CD147) in multiple myeloma. Hematology 21, 26-33. doi: 10.1179/1607845415Y.0000000043

Wardlaw, J. M., Smith, C., and Dichgans, M. (2019). Small vessel disease: mechanisms and clinical implications. Lancet Neurol. 18, 684-696. doi: 10.1016/ S1474-4422(19)30079-1

Wardlaw, J. M., Smith, E. E., Biessels, G. J., Cordonnier, C., Fazekas, F., Frayne, R., et al. (2013). Neuroimaging standards for research into small vessel disease and its contribution to ageing and neurodegeneration. Lancet Neurol. 12, 822-838. doi: 10.1016/S1474-4422(13)70124-8

Yuan, Y. J., Xu, K., Luo, Q., and Yu, J. L. (2014). Research progress on vertebrobasilar dolichoectasia. Int. J. Med. Sci. 11, 1039-1048. doi: 10.7150/ijms. 8566

Zhai, F. F., Yan, S., Li, M. L., Han, F., Wang, Q., Zhou, L. X., et al. (2018). Intracranial arterial dolichoectasia and stenosis: risk factors and relation to cerebral small vessel disease. Stroke 49, 1135-1140. doi: 10.1161/STROKEAHA. 117.020130

Zhang, M., Zhu, W., Yun, W., Wang, Q., Cheng, M., Zhang, Z., et al. (2015). Correlation of matrix metalloproteinase-2 single nucleotide polymorphisms with the risk of small vessel disease (SVD). J. Neurol. Sci. 356, 61-64. doi: 10.1016/j.jns.2015.04.056

Zhu, Y. Q., Xing, H., Dai, D., Kallmes, D. F., and Kadirvel, R. (2017). Differential interstrain susceptibility to vertebrobasilar dolichoectasia in a mouse model. AJNR Am. J. Neuroradiol. 38, 611-616. doi: 10.3174/ajnr.A5028

Conflict of Interest Statement: The authors declare that the research was conducted in the absence of any commercial or financial relationships that could be construed as a potential conflict of interest.

Copyright (C) 2019 Zhang, Peng, Zhang, Ma, Zhao, Yin and Wei. This is an openaccess article distributed under the terms of the Creative Commons Attribution License (CC BY). The use, distribution or reproduction in other forums is permitted, provided the original author(s) and the copyright owner(s) are credited and that the original publication in this journal is cited, in accordance with accepted academic practice. No use, distribution or reproduction is permitted which does not comply with these terms. 